\title{
Alert System for Driver Drowsiness using Real Time detection
}

\author{
Aman Dohare \\ I.T. Final year \\ Department of Information \\ Technology \\ Rajkiya Engineering College \\ Ambedkarnagar, U.P., India
}

\author{
Gargie Bharti \\ I.T. Final year \\ Department of Information \\ Technology \\ Rajkiya Engineering College \\ Ambedkarnagar, U.P., India \\ Dr. Sudhakar Tripathi \\ Head of Dapartement of Information Technology \\ Rajkiya Engineering College \\ Ambedkarnagar,U.P., India
}

\author{
Amit Kumar \\ Assistant Profrssor \\ Department Information \\ Technology \\ Rajkiya Engineering College \\ Ambedkarnagar, U.P. India
}

\begin{abstract}
Various drivers drive their vehicle, transport, truck, merchandise vehicle, movers during day and getting dull, and a few of the time, they experience the evil impacts of absence of rest reliable with different investigations and reports, weakness and tiredness are a number of the most sources of serious street mishaps. The elemental motivation behind the framework is to detect the driver's facial condition, and in case the most thrust is feeling tired, at that time, the framework will trigger an admonition yield inside such a caution the threat ringer or educate the travelers. This calculation camera ceaselessly records the facial behavioral milestone and development of eyes and lips of the most thrust. Due to the consideration conclusion period for lethargic drivers are longer than ordinary eye flickering. Through that live video spilling, a foothold is extricated for picture preparing. Pictures are caught ordinarily at a fix outline pace of $20 \mathrm{fps}$. Utilizing the image and comment informational collection, the framework comprehends the position the most thrust is feeling languid by estimating the directions of the right and left eye, nose, mouth, left and right ear temple. The human visual setting how or another speaks to the element of the scene with two or three significant data in it. Using Python and two or three OpenCV libraries, the estimation picks unequivocal, perceiving bits of data with reference to a person's face and concentrate those major factor structure the photos. For the premier part, it takes to diminish photos of the article as data, and a brief time later perceives the driver's condition snared in to an ebb and flow condition for sluggishness using the consideration edge extent. At the purpose, when the structure recognizes any sporadic improvement of the most thrust, it'll alert the most thrust, explorer, or naval force controller quite caution, notice popups, vibration, at that time forward.
\end{abstract}

\section{INTRODUCTION}

Drowsy methods sluggish and having low vitality. Drowsiness is position of on the brink of rest, a strong urge for rest. Drowsiness alludes to be unable to stay your eyes open, or feeling tired or tired. Sleepiness, additionally called abundance languor. It may prompt carelessness or nodding off at unseemly occasions. It tends to be joined by shortcoming, laziness, and absence of mental sharpness. Discouragement, distress and stress are additionally connected with traded off rest. Presently drowsiness of individual driving vehicle is critical. It's not going to be due to some clinical issue yet long driving by a drained driver. This might cause drowsiness so there's a requirement to spot this to take care of a vital good ways from miss occurring components are specified for 3 reasons: (1) basic use when organizing singular papers, (2) modified consistence to electronic prerequisites that empower the synchronous or later creation of electronic items, and (3) similarity of favor all through a meeting procedures. Edges, segment widths, line separating, and sort styles are implicit; examples of the sort styles are given all through this report and are recognized in an italic kind, inside brackets, following the occurrence. Some components, like multi-leveled equations, designs, and tables aren't, It prescribed, although the varied table text styles are given.The formatted will get the chance to make these parts, fusing the relevant standards that follow ease not that they are not perfecting.

\subsection{Driver Fatigue and Road Accidents}

Driver exhaustion once in a while brings about street mishaps consistently. It is difficult to gauge the specific measure of rest related mishaps however research presents that driver exhaustion might be a contributing explanation in up to $20 \%$ in street mishaps. These sorts of mishaps are about half increasingly expected to bring about death or genuine hurt. They happen mostly at higher speed impacts. What's more, the driver who has nodded off can't slow down. Drowsiness decreases reaction time which is a genuine component of secure driving. It likewise lessens readiness, cautiousness, and focus with the goal that the ability to perform consideration based exercises for example driving is hindered. The speed at which data is handled is additionally diminished by drowsiness. The nature of dynamic may likewise be influenced. Unmistakably drivers know when they are feeling lethargic, thus settle on a cognizant choice about whether to keep driving or to stop for a rest. It might be that the individuals who continue driving belittle the danger of really nodding off while driving. Or on the other hand it might be that a few drivers decide to disregard the dangers in the manner drivers drink. Accidents brought about by tired drivers are destined to occur on long excursions on repetitive streets, for example, motorways, somewhere in the range of $2 \mathrm{pm}$ and $4 \mathrm{pm}$ 
particularly in the wake of eating or taking a mixed beverage, somewhere in the range of $2 \mathrm{am}$ and $6 \mathrm{am}$, in the wake of having less rest than typical, in the wake of drinking liquor, it driver takes meds that cause drowsiness and after long working hours or on ventures home after long moves, particularly night shifts.

Driver drowsiness is one of the major causes Sluggishness and exhaustion can frequently influence an individual's driving capacity some time before he/she even notification that he/she is getting drained. Weariness related accidents are regularly more extreme than others since driver's response times are postponed or the drivers have neglected to make any man oeuvres to maintain a strategic distance from an accident. The quantity of hours spent driving has a solid relationship to the quantity of weakness related mishaps.

\subsection{Motive of Detection of Problem}

Driver drowsiness is a genuine risk in transportation frameworks. It has been recognized as a'n immediate or contributing reason for street mishap. Drowsiness can truly slow response time, decline mindfulness and weaken a driver's judgment. It is inferred that driving while drowsy is like driving affected by liquor or medications. In industrialized nations, drowsiness has been assessed to be associated with $2 \%$ to $23 \%$ everything being equal.

Frameworks that distinguish when drivers are turning out to be drowsy and sound an admonition guarantee to be a significant guide in forestalling mishaps. Conceivable procedure for distinguishing drowsiness in drivers can be commonly separated into the accompanying classifications: detecting of physiological qualities, detecting of driver activity, detecting of vehicle reaction, observing the reaction of driver. Driver weariness is a noteworthy factor in an enormous number of vehicle mishaps. Ongoing insights gauge that yearly 1,200 passings and 76,000 wounds can be credited to exhaustion related accidents. The advancement of advances for identifying or forestalling drowsiness in the driver's seat is a significant challenge in the field of mishap shirking frameworks. On account of the risk that drowsiness presents out and about, strategies should be created for balancing its effects.

\subsection{Aim of the Project}

The aim of this project is to build up a drowsiness identification framework. The spotlight will be put on structuring a framework that will precisely screen the open or shut condition of the driver's eyes progressively. By checking the eyes, it is accepted that the manifestations of driver weariness can be distinguished early enough to evade a fender bender. Location of weakness includes the perception of eye developments and flicker designs in an arrangement of pictures of a face. In this undertaking we build up a framework that can precisely distinguish languid driving and make alerts in like manner, which keep the drivers from drowsy driving and make a more secure driving condition.

Functions and Features

This framework has numerous highlights that make it special and practical. These highlights include:
1.Eye extraction, utilize open and near decide sluggishness

2.Daytime and night discovery

3.Real time picture preparing and identification

4.Sound admonition framework to redraw driver's consideration

5.Little surmising and expected danger to driver's ordinary driving.

Feeling tired while driving could cause perilous car crash. Nonetheless, when driving alone on expressway or driving over an extensive stretch of time, drivers are slanted to exhausted and feel tired, or even nod off. These days the greater part of the results of driver against rest discovery sold in the market is basically headphone making discontinuous clamors, which is very irritating and wasteful. In that capacity, there is a popularity for inexpensively effective driver rest discovery. Hence, we thought of a plan to build up a driver hostile to rest alert framework, which could adequately satisfy this need.

\subsection{Objectives}

The goals of this task are to build up a drowsiness recognition framework that can recognize drowsy or weariness in drivers to forestall mishaps and to improve security on the streets. This framework capable precisely screens the open or shut condition of the driver's eye. There are loads of goals for the Drowsy Driver Detection framework as following:

1. Lethargy identification is effective and cautions will be produced just when requested.

2. Upgraded calculation to guarantee the murkiness recognition capacity

3. Little deduction and possible peril to driver's ordinary driving

\subsection{Scope}

The Scope of the Drowsy Driver Detection framework in the Morden period Drowsy Driver Detection framework diminishes the vehicle street mishap and furthermore this framework utilized for security reason for a driver. Scope of Drowsy Driver Detection system:-

1. Reduce car accident

2. Security purpose of the driver

\section{ANALYSIS \\ 2.1 System Requirement Analysis}

Framework improvement needs to significant parts. Framework investigation and framework plan. Framework examination and configuration alludes to the way toward looking at a business circumstance with the purpose of improving it through better techniques and system. Framework configuration is the way toward arranging another business framework to supplant the old. In any case, before this arranging should be possible, we should altogether comprehend the old framework and decided how the PC can be best used to make its activity progressively successful.

Framework examination is procedure of understanding the current framework by social occasion and deciphering realities, diagnosing issues, and utilizing the realities to 
improve the current framework. This is the activity of framework investigator. Having decided the necessities and what is to be de framework planner in the new framework remembering the target set during the framework examination

\subsubsection{Technology Specification}

\section{System Feasibility:-}

The expression "requirement for speed" is the response for the above inquiry. The framework gives such offices, which make its taking care of/working quicker just as alluring. Proposed framework furnishes with following arrangements:

1. It gives "better and productive" administration to individuals.

2. Reduce Car Road mishap.

\subsection{Process Model used}

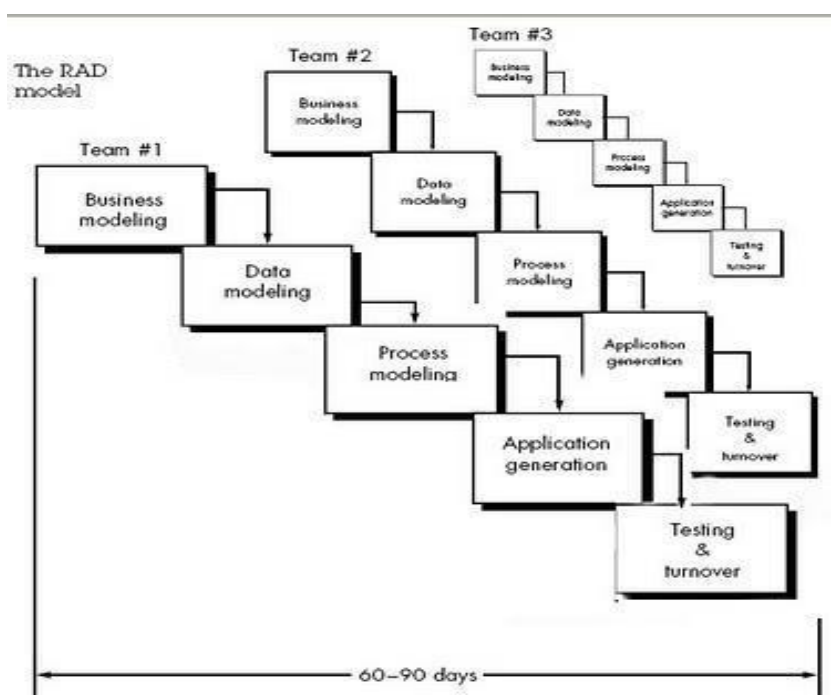

Description about RAD model

Rapid Application development (RAD) is a consistent programming development process model that anxieties a short development cycle (somewhere in the range of 60-90 days). The RAD model is a fast adjustment of the direct successive model/Waterfall model.This model relies upon prototyping and iterative improvement with no particular arranging included. The way toward composing the product itself includes the arranging required for building up the item. This should be utilized when there is a need to make a framework that can be modularized in 2-3 months of time. It ought to be utilized if there's high accessibility of originators for displaying and the spending plan is sufficiently high to manage the cost of their expense alongside the expense of mechanized code producing devices.

The RAD approach encompasses the following phases:-

\section{Business Modelling}

The data stream among business capacities is demonstrated such that responds to the accompanying inquiries:

What data drives the business forms?

What data is created? Who produces it?

Where does the data stream? Who forms it?

\section{Data Modelling}

The data stream characterized as a feature of the business displaying stage is refined into a lot of information protests that are expected to help the business. The qualities called traits of each items are recognized and the connections between the articles are then characterized.

\section{Process Modelling}

The information objects characterized in the information demonstrating stage are changed to accomplish the data stream important to actualize the business capacities. Preparing depictions are made for including, changing, erasing or recovering an information object.

\section{Application Generation}

RAD accept the utilization of fourth era methods. As opposed to making programming utilizing ordinary third era programming dialects,

RAD process attempts to utilize the mechanized apparatuses to encourage the development of the product.

\section{Testing and Turnover}

Since the RAD forms accentuate reuse, a large number of the program parts have just been tried. This spares time, cash andthe general chance to test an application additionally lessens impressively.

\subsection{Informal Flow Representation}

UML Diagram:-

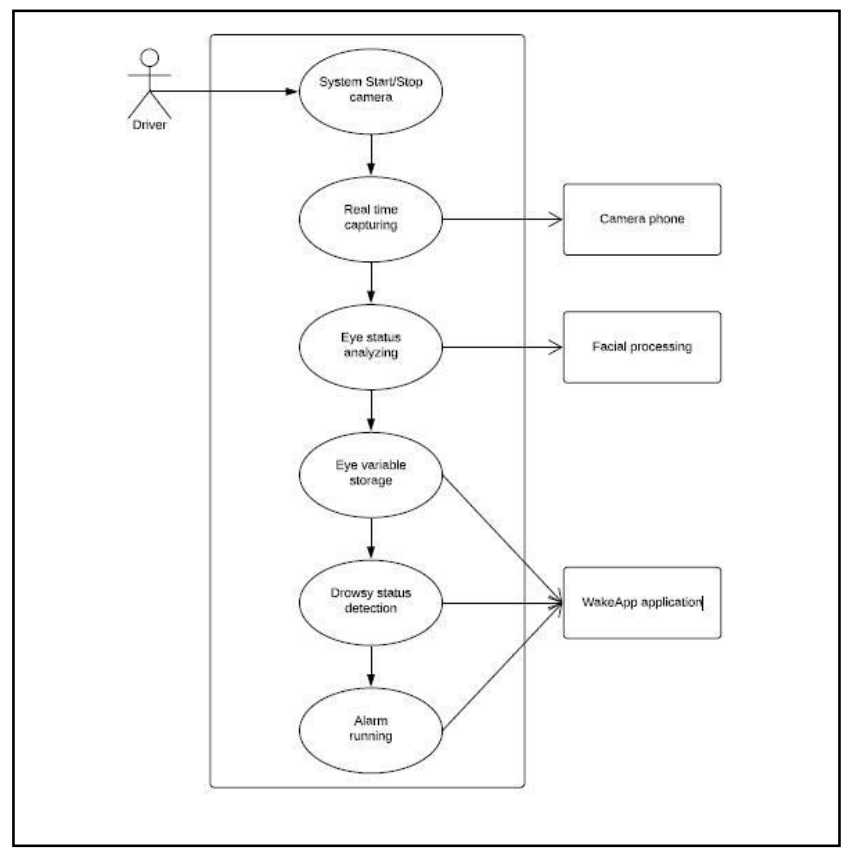

Fig: 2.3.1 UML Diagram

\section{PLANNING}

3.1 Software Project Estimation

Programming Estimation Best Practices, Tools, and Techniques covers all aspects of programming estimation. It gives a point by point clarification of the different strategies for assessing programming size, development exertion, cost, 
and timetable, including a far reaching clarification of test exertion estimation. Stressing that product estimation ought to be founded on very much characterized forms, it presents programming estimation best practices and tells the best way to stay away from basic traps. This guide offers bearing on which strategies are generally suitable for every one of the diverse undertaking types normally executed in the product development space and models for choosing programming estimation instruments. It subtleties venture planning, including asset leveling and the idea of profitability, as pertinent to programming estimators, showing the numerous advantages of moving from the current large scale efficiency way to deal with a miniaturized scale profitability in programming estimation. It gives the important direction expected to evaluate the expense and time required to finish programming ventures inside a sensible safety buffer for viable programming development.

\subsection{Timeline Chart}

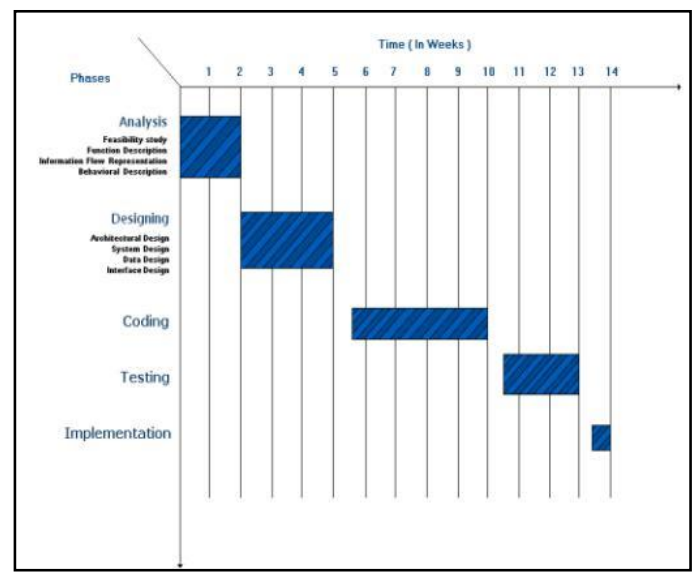

Fig 3.2: Timeline Chart

\subsection{Resource Planning}

In this project we used a Webcam and laptop which use to run an algorithm.

\subsubsection{Software Requirement}

\begin{tabular}{|l|l|}
\hline Category & Name \\
System & Any Mac/windows/Ubuntu \\
\hline Tool & Vs code, Vlc Player, Webcam \\
\hline Technology & Python(ML) \\
\hline Library & Open Cv, Scipy, Numpy, Scikit- \\
& image,etc. \\
\hline
\end{tabular}

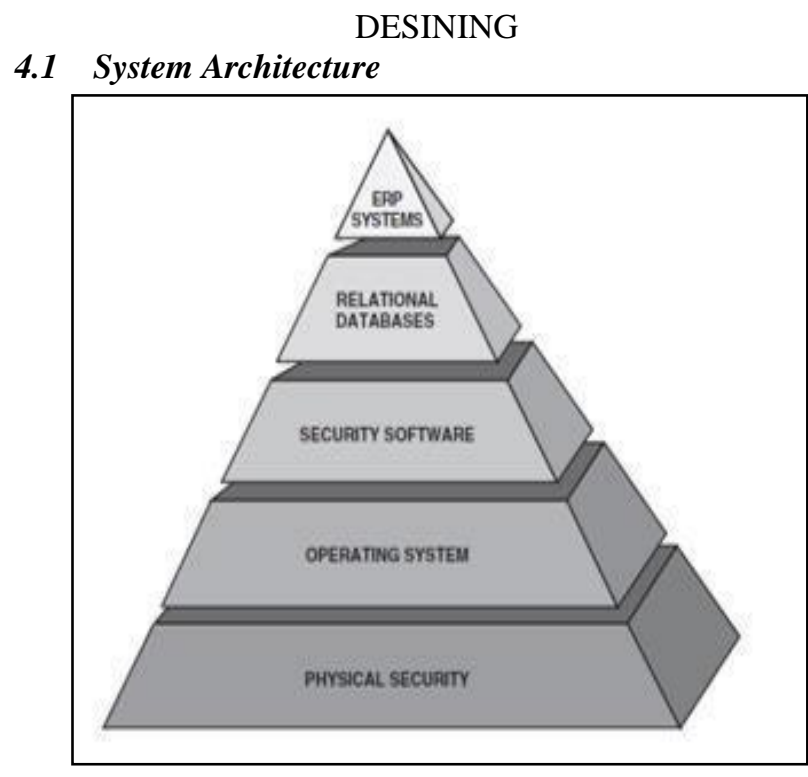

1. A plan should display a building structure that:

1. Has been made utilizing unmistakable structure designs.

2. Is made out of segments that display great structure attributes.

3. Can be executed in a developmental manner, in this way encouraging Implementation and testing.

2. A plan ought to be particular; that is, the product ought to be sensibly apportioned into components that perform explicit capacities and sub capacities.

3. A plan ought to contain unmistakable portrayals of information, design, Interfaces and parts (modules).

4. A plan should prompt information structures that are suitable for the items to be actualized and are drawn from conspicuous information designs.

5. A structure should prompt segments that display autonomous practical qualities.

6. A plan should prompt interfaces that diminish the multifaceted nature of associations among modules and with the outer condition.

7. A plan ought to be determined utilizing a repeatable technique that is driven by data got during programming prerequisites examination.

\subsection{Project Life cycle}

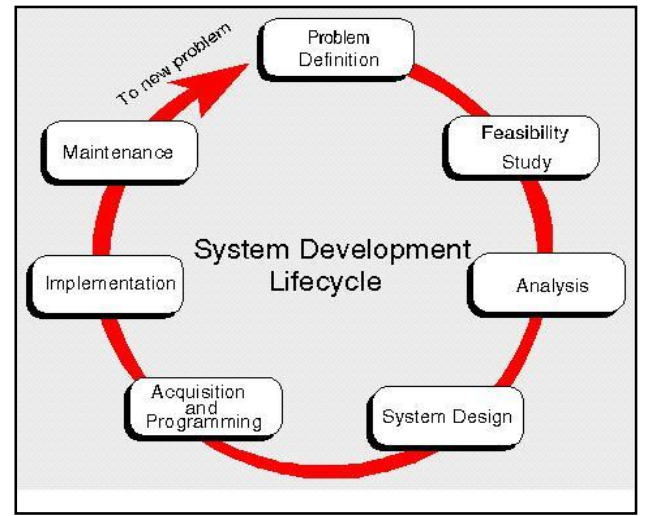

Fig 4.2: Project Life cycle 


\section{TECHNOLOGY \\ 5.1 Language used for the implementation Python}

\subsubsection{What is Python?}

Python is a ground-breaking present day PC programming language. It bears a few likenesses to FORTRAN, one of the soonest programming dialects, however it is substantially more remarkable than FORTRAN. Python permits you to utilize factors without proclaiming them (i.e., it decides types verifiably), and it depends on space as a control structure. You are not compelled to characterize classes in Python (in contrast to Java) however you are allowed to do so when advantageous. Python was created by Guido van Rossum, what's more, it is free programming. Free as in - free beer,\| in that you can acquire Python without going through any cash. However, Python is likewise free in other significant manners, for instance you are allowed to duplicate it the same number of times as you like, and allowed to examine the source code, and make changes to it. There is an overall development behind free programming, started in 1983 by Richard Stallman. This report centers around learning Python to do numerical computations. We expect the peruser has some information on fundamental science, however we make an effort not to accept any past introduction to PC programming, albeit whatever presentation would absolutely be useful. Python is a decent decision for numerical figurings, since we can compose code rapidly, test it effectively, and its language structure is like the manner in which scientific thoughts are communicated in the scientific writing. By learning Python you will likewise be learning a significant device utilized by many web engineers.

\subsubsection{Installation and Documentation}

Mac OS X or Linux, then Python should already be installed on your computer by default. If not, you can download the most recent adaptation by visiting the Python landing page, at http://www.python.org where you will likewise discover heaps of documentation and other valuable data. Windows clients can likewise download Python at this site.

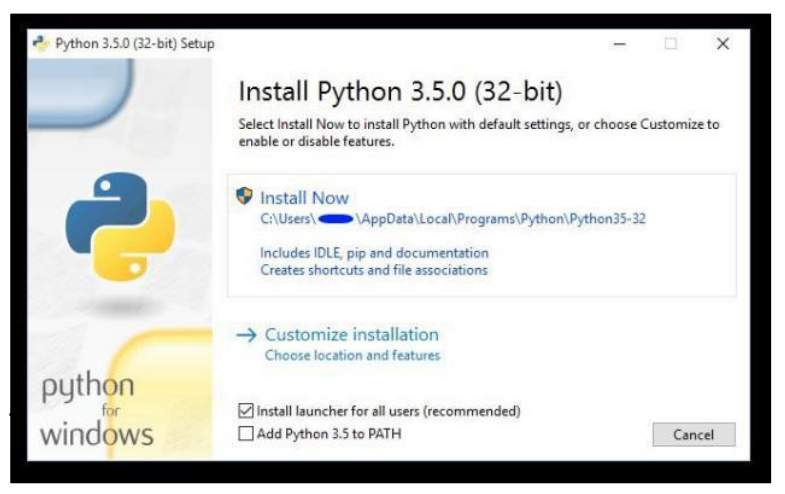

\subsubsection{Features of Python}

\begin{abstract}
Simple
Python is a straightforward and moderate language. Perusing a decent Python program feels practically like understanding English, albeit exceptionally exacting English! This pseudo-code nature of Python is probably the best quality. It permits you to focus on the answer for the issue as opposed to the language itself.
\end{abstract}

\section{Easy to Learn}

As you will see, Python is very simple to begin with. Python has a phenomenally basic grammar, as of now referenced.

\section{Free and Open Source}

Python is a case of FLOSS (Free/Libré and Open Source Software). In basic terms, you can unreservedly circulate duplicates of this product, read its source code, make changes to it, and use bits of it in new free projects. FLOSS depends on the idea of a network which shares information. This is one reason why Python is so acceptable. It has been made and is continually improved by a network who simply need to see a superior Python.

\section{High-level Language}

At the point when you compose programs in Python, you never need to make a fuss over the low-level subtleties, for example, dealing with the memory utilized by your program, and so on.

\section{Object Oriented}

Python bolsters method arranged programming just as article situated programming. In strategy situated dialects, the program is worked around systems or capacities which are only reusable bits of projects. In object-situated dialects, the program is worked around objects which join information and usefulness. Python has an extremely ground-breaking yet shortsighted method of doing OOP, particularly when contrasted with large dialects like $\mathrm{C}++$ or Java.

\section{Extensive Libraries}

The Python Standard Library is immense for sure. It can assist you with doing different things including normal articulations, documentation age, unit testing, stringing, databases, internet browsers, CGI, FTP, email, XML, XMLRPC, HTML, WAV documents, cryptography, GUI (graphical UIs), and other framework subordinate stuff.

\subsubsection{NumPy}

NumPy is the basic bundle for logical processing in Python. It is a Python library that gives a multidimensional cluster object, different determined items, (for example, conceal exhibits and frameworks), and a variety of schedules for quick procedure on exhibits, including scientific, legitimate, shape control, arranging, choosing, I/O, discrete Fourier changes, essential straight polynomial math, fundamental measurable tasks,arbitrary recreation and significantly more. At the center of the NumPy bundle, is the ND cluster object. This embodies $\mathrm{n}$-dimensional varieties of homogeneous information types, with numerous activities being acted in gathered code for execution. 
There are a few significant contrasts between NumPy exhibits and the standard Python successions:

NumPy exhibits have a fixed size atcreation, not at all like Python records (which can develop progressively). Changing the size of a ndarraywill make another exhibit and erase the first.

The components in a NumPy exhibit are totally required to be of similar information type, and in this way will be a similar size in memory. The special case: one can have varieties of (Python, including NumPy) objects, consequently taking into account varieties of various measured components.

NumPy clusters encourage progressed scientific and different sorts of procedure on enormous quantities of information. Ordinarily, such tasks are executed more effectively and with less code than is conceivable utilizing Python's worked in successions.A developing plenty of logical and numerical Python-based bundles are utilizing NumPy clusters; however these normally bolster Pythonarrangement input, they convert such contribution to NumPy exhibits preceding handling, and they frequently yield NumPy clusters. At the end of the day, so as to proficiently utilize a lot(maybe even a large portion) of the present logical/scientific Python-based programming, simply realizing how to utilize Python's worked in succession types is inadequate - one likewise has to realize how to utilize NumPy exhibits.

\subsubsection{Matplotlib}

Matplotlib is a library for making 2D plots of exhibits in Python. In spite of the fact that it has its inceptions in copying the MATLAB designs orders, it is free of MATLAB, and can be utilized in a Pythonic, object situated way. Despite the fact that Matplotlib is composed principally in unadulterated Python, it utilizes NumPy and other augmentation code to give great execution even to huge clusters. Matplotlib is structured with the way of thinking that you ought to have the option to make basic plots with only a couple of orders, or only one! On the off chance that you need to see a histogram of your information, you shouldn't have to start up objects, call strategies, set properties, etc; it should simply work.

For quite a long time, I used to utilize MATLAB only for information examination and perception. MATLAB exceeds expectations at making decent looking plots simple. At the point when I started working with EEG information, I found that I expected to compose applications to connect with my information, and built up an EEG examination application in MATLAB. As the application developed in multifaceted nature, cooperating with databases, http servers, controlling complex information structures, I started to strain against the constraints of MATLAB as a programming language, and chose to begin once again in Python. Python more than compensates for the entirety of MATLAB's lacks as a programming language, yet I was experiencing issues finding a 2D plotting bundle.Matplotlib is utilized by numerous individuals in a wide range of settings. A few naturally produce PostScript documents to send to a printer or distributers. Others convey Matplotlib on a web application server to produce PNG yield for incorporation in progressively created website pages. Some utilization Matplotlib intuitively from the Python shell in Tkinter on Windows.

\subsection{Library used for the implementation}

\section{OpenCV}

\subsubsection{What is OpenCV?}

OpenCVis an open source (see

http://opensource.org) PC vision library available from http://SourceForge.net/ventures/opencvlibrary. OpenCV was intended for computational productivity and having a high spotlight on constant picture location. OpenCV is coded with advanced $\mathrm{C}$ and can take work with multicore processors. In the event that we want progressively programmed enhancement utilizing Intel models. [Intel], you can purchase Intel's Integrated Performance Primitives (IPP) libraries [IPP]. These comprise of low-level schedules in different algorithmic territories which are improved. OpenCV consequently utilizes the IPP library, at runtime if that library is introduced. One of OpenCV objectives is to give an easy to-utilize PC vision foundation which causes individuals to fabricate exceptionally refined vision applications quick. The OpenCV library, containing more than 500 capacities, ranges numerous regions in vision. Since PC vision and AI oft en goes connected at the hip, OpenCV additionally has a total, broadly useful, Machine Learning Library (MLL). This sub library is centered around factual example acknowledgment and bunching. The MLL is helpful for the vision capacities that are the premise of OpenCV's value, however is sufficiently general to be utilized for any AI issue.

\subsubsection{What Is Computer Vision?}

$\mathrm{PC}$ vision is the changing of information from a still, or camcorder into either a portrayal or another choice. Every such change are performed to accomplish a specific objective. A PC gets a matrix of numbers from a camera or from the circle, and that's all there is to it. For the most part, there is no worked in design acknowledgment or programmed control of center and gap, no cross-relationship with long stretches of understanding. Generally, vision frameworks are still decently guileless.

\subsubsection{The Origin of OpenCV}

OpenCV came out of an Intel Research activity intended to propel CPU-escalated applications. Toward this end, Intel propelled different tasks that included ongoing beam following and furthermore 3D show dividers. One of the software engineers working for Intel at the time was visiting colleges. He saw that a couple of top college gatherings, similar to the MIT Media Lab, used to have all around created just as inside open PC vision frameworks-code that was passed starting with one understudy then onto the next and which gave each ensuing understudy a significant establishment while building up his own vision application. Rather than reexamining the essential capacities from starting, another understudy may begin by adding to that which came previously. 


\subsubsection{OpenCV Structure and Content}

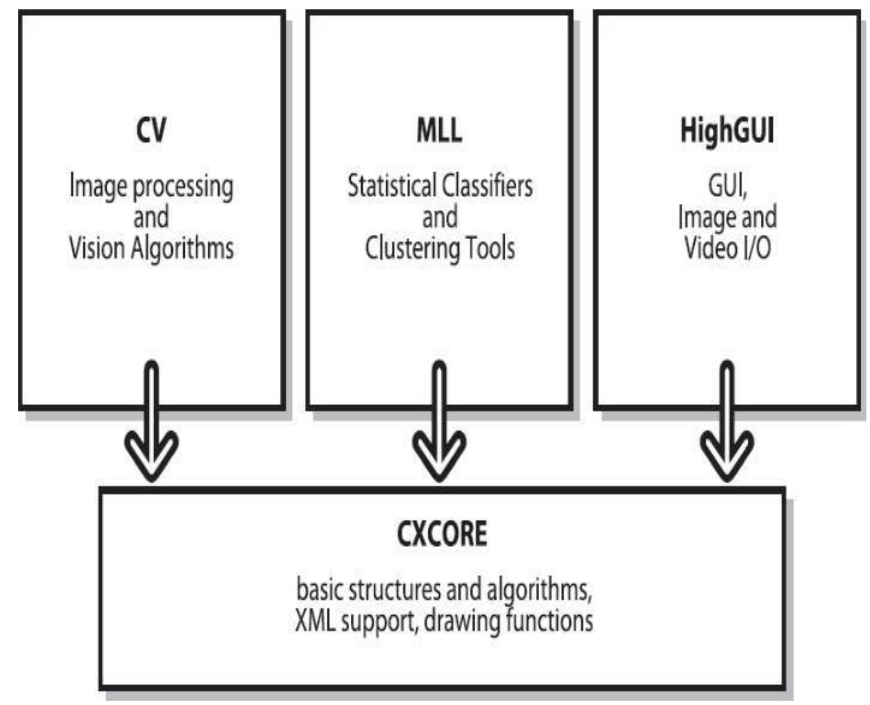

OpenCV can be comprehensively organized into five essential segments, four of which are appeared in the figure. The CV part contains mostly the essential picture preparing and more elevated level PC vision calculations; MLL the AI library incorporates numerous measurable classifiers just as grouping devices. High GUI segment contains I/O schedules with capacities for putting away, stacking video and pictures, while CXCore contains all the essential data structure and content.

\subsubsection{Why OpenCV?}

Specific

OpenCV was intended for picture handling. Each capacity and information structure has been planned in view of an Image Processing application. In the mean time, Matlab, is very nonexclusive. You can get nearly everything on the planet by methods for tool stash. It might be money related tool compartments or particular DNA tool kits.

Efficient

Matlab utilizes just an abundant excess framework assets. With OpenCV, we can pull off as meager as $10 \mathrm{mb}$ RAM for a constant application. In spite of the fact that with the present PCs, the RAM factor is certainly not a major thing to be stressed over. In any case, our sleepiness discovery framework is to be utilized inside a vehicle in a manner that is non-meddlesome and little; so a low handling prerequisite is fundamental.

In this manner we can perceive how OpenCV is a superior decision than Matlab for a continuous laziness identification framework.

Speedy

Matlab is simply excessively moderate. Matlab itself was based upon Java. Additionally Java was based upon C. So when we run a Matlab program, our PC gets caught up with attempting to decipher and arrange all that confounded Matlab code. At that point it is transformed into Java, lastly executes the code.
On the off chance that we use $\mathrm{C} / \mathrm{C}++$, we don't burn through such time. We legitimately give machine language code to the PC, and it gets executed. So at last we get more picture preparing, and not more deciphering.

In the wake of doing some ongoing picture preparing with both Matlab and OpenCV, we normally got exceptionally low speeds, a limit of around 4-5 casings being handled every second with Matlab. With OpenCV be that as it may, we get genuine continuous handling at around 30 edges being prepared every second.

\section{Machine Learning}

\subsubsection{What Is Machine Learning?}

The objective of AI is to transform information into data. Subsequent to having gained from a social event of information, we need a machine that can respond to any question about the information:

1. What are different information that are comparative to given information?

2. Is there a face in the picture?

3. What sort of promotion will impact the client?

\subsubsection{OpenCV ML Algorithms}

The AI calculations that are remembered for OpenCV are given as follows. All the calculations are available in the ML library separated from Mahalanobis and K-means, which are available in CVCORE, and the calculation of face recognition, which is available in $\mathrm{CV}$.

\section{Mahalanobis}

It is a proportion of separation that is liable for the stretchiness of the information. We can isolate out the covariance of the offered information to locate this out. If there should be an occurrence of the covariance being the personality network (for example indistinguishable difference), this measure will be indistinguishable from the Euclidean separation.

\section{K-means}

It is an unaided grouping calculation which connotes a conveyance of information w.r.t. $\mathrm{K}$ focuses, $\mathrm{K}$ being picked by the coder. The contrast between K-means and desire expansion is that in K-means the focuses aren't Gaussian. Likewise the bunches framed look to some degree like cleanser rises, as focuses contend to possess the nearest information focuses. All these group zones are generally utilized as a type of inadequate histogram container for speaking to the information.

\section{Normal/Naïve Bayes classifier}

It is a generative classifier where highlights are frequently thought to be of Gaussian conveyance and additionally measurably autonomous from each other. This supposition that is generally bogus. That is the reason it's generally known as a -naïve Bayes\| classifier. All things considered, this strategy as a rule works shockingly well. 


\section{Decision trees}

It is a discriminative classifier. The tree basically finds a solitary information highlight and decides a limit estimation of the current hub which best partitions the information into various classes. The information is broken into parts and the strategy is recursively rehashed through the left just as the correct parts of the decision tree. Regardless of whether it isn't the top entertainer, it's typically the primary thing we attempt as it is quick and has a high usefulness.

\section{Boosting}

It is a discriminative gathering of classifiers. In boosting, the last order decision is made by taking into account the joined weighted arrangement decisions of the gathering of classifiers. We learn in preparing the gathering of classifiers consistently. Every classifier present in the gathering is known as a weak classifier. These weak classifiers are generally made out of single-variable decision trees known as stumps. Taking in its arrangement decisions from the given information and furthermore learning a load for its vote dependent on its precision on the information are things the decision tree gets the hang of during preparing. While every classifier is prepared in a steady progression, the information focuses are re-weighted to make more consideration be paid to the information focuses in which mistakes were made. This proceeds until the net blunder over the whole informational index, acquired from the joined weighted vote of all the choice trees present, falls under a specific edge. This calculation is generally powerful when a huge amount of preparing information is accessible.

\section{Random trees}

It is a discriminative backwoods of a great deal of choice trees, every one of which is worked down to a maximal parting profundity. At the hour of learning, each hub of each tree is permitted a decision 20 of parting factors, however just from a randomly produced subset of the considerable number of information highlights. This guarantees all the trees become factually autonomous and a leader. In the run mode, all the trees get an unweighted vote. Random trees are normally very powerful. They can likewise perform relapse by taking the normal of the yield numbers from each tree.

\section{Face detector (Haar classifier)}

It is an article recognition application. It depends on a savvy utilization of boosting. A prepared frontal face detector is accessible with the OpenCV dissemination. This works remarkably well. We can prepare the calculation for different articles by utilizing the product gave. This works superbly for unbending articles with trademark sees.

\section{Expectation maximization (EM):}

It is utilized for bunching. It is a generative solo calculation it fits $\mathrm{N}$ multidimensional Gaussians to the information, $\mathrm{N}$ being picked by the client. It can go about as a productive path for speaking to a progressively perplexing appropriation utilizing just a couple of boundaries (for example means and differences).Generally utilized in division, it very well may be contrasted and K-means.

\subsubsection{Variable Importance}

This is the significance of a specific variable in a dataset. One of the employments of variable significance is for diminishing the quantity of highlights the classifier needs to consider. In the wake of beginning with various highlights, we train our classifier to discover the significance of each element comparable to the various highlights. We at that point dispose of immaterial highlights. Dispensing with insignificant highlights helps in improving pace execution (since it can wipe out the preparing taken for registering those highlights) and furthermore makes preparing and testing quicker. At the point when we don't have adequate information, which is consistently the situation, at that point wiping out irrelevant factors helps in expanding order precision, which thus yields quicker preparing and better outcomes.

Biermann's calculation for variable significance is as per the following

1. A classifier is prepared on the preparation set.

2. A approval or test set is utilized for deciding the precision of the classifier.

3. For every information point and a picked highlight, another incentive for that component is randomly browsed among the qualities that the element has in the remainder of the informational collection (known as sampling with replacement).This helps in guaranteeing that the appropriation of that component remains equivalent to in the first informational index, nonetheless, the real structure or significance of that element is removed (as its worth is picked at random from the remainder of the information).

4. The classifier is prepared on the adjusted arrangement of the preparation information and afterward the exactness of order estimated on the changed test or approval informational collection. When randomizing of an element hurt precision a great deal, at that point it is suggested that the element is imperative. When randomizing of a component doesn't do any harm exactness much, at that point the element is of little significance and is a reasonable contender for removal.

5. The unique test or approval informational collection is reestablished and the following element is attempted until we are done. The outcome acquired requests each element by its significance. This methodology utilized above is incorporated with random trees and choice trees and boosting. In this manner, we can utilize these calculations to choose which factors we will at long last use as highlights; at that point we can utilize the enhanced element vectors to prepare the classifier.

\subsubsection{Object Detection}

The Viola-Jones cascade is a binary classifier:

It just chooses whether or not the item in a picture is like the preparation set. Any picture that doesn't contain the object of intrigue can be transformed into a negative example. It is 
valuable to take the negative pictures from a similar sort of information. That is, on the off chance that we need to learn faces in online recordings, for best outcomes we should take our negative examples from equivalent casings .However, similar outcomes can at present be gotten utilizing negative examples taken from some other source. Again we put the pictures into at least one registries and afterward make a list document comprising of a rundown of picture filenames, one for every line. For instance, a picture list document called background.idx.

\subsection{Block Diagram}

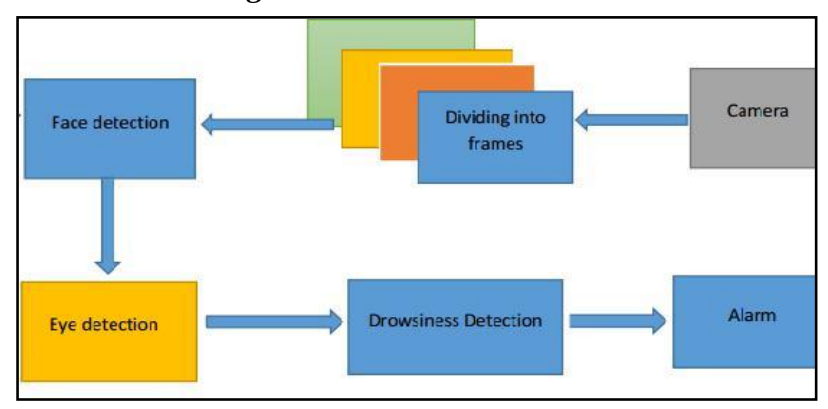

Sleepiness of an individual can be estimated by the allencompassing timeframe for which his/her eyes are in shut state. In our framework, essential consideration is given to the quicker recognition and preparing of information. The quantity of edges for which eyes are shut is observed. On the off chance that the quantity of edges surpasses a specific worth, at that point an admonition message is created on the presentation indicating that the driver is feeling sluggish. In our calculation, first the image is obtained by the webcam for handling. At that point we utilize the dataset to distinguish the countenances in every individual edge. On the off chance that no face is distinguished, at that point another edge is gained. In the event that a face is identified, at that point an area of enthusiasm for set apart inside the face. This locale of intrigue contains the eyes. Characterizing a district of intrigue essentially lessens the computational prerequisites of the framework. After that the eyes are distinguished from the district of enthusiasm by utilizing dataset Euclidian spaces point.

\subsection{Image Acquisition}

The capacity cvCaptureFromCAM distributes and instated the CvCapture structure for perusing a video stream from the camera.

CvCapture* cvCaptureFromCAM (int record);

Record of the camera to be utilized. In the event that there is just a single camera or it doesn't make a difference what camera to utilize, - 1 might be passed.

CvSetCaptureProperty Sets camera properties for instance cvSetCaptureProperty (outline $=$

imutils.resize (outline, width=450));

The capacity cvQueryFrame () gets an edge from a camera or video document,

The capacity cvQueryFrame () gets an edge from a camera or video document, decompresses it and brings it back. This capacity is only a blend of Grab Frame and Retrieve Frame, however in one call. The returned picture ought not be discharged or adjusted by the client. In case of a mistake, the arrival worth might be NULL.

\subsection{Dividing into Frames}

We are managing ongoing circumstance where video is recorded and must be handled. In any case, the preparing or utilization of calculation should be possible just on a picture. Subsequently the caught video must be isolated into frames for breaking down.

\subsection{Face Detection}

In this stage we identify the area containing the essence of the driver. A predetermined calculation is for location of face in each edge. By face discovery we implies that finding the face in a casing or as such discovering area of facial characters through a kind of innovation with the utilization of PC. The casing might be any irregular edge. Just facial related structures or highlights are distinguished and all others sorts of articles like structures, tree, bodies are overlooked.

We realize that face is likewise a sort of item. So we can think about location of face as a specific instance of item discovery. In this sort of item kind of class recognition, we attempt to know where the articles in the intrigue picture are found and what is their size which may has a place with a specific class. Crafted by calculation that is made for face location is generally focused on finding the front side of the face. In any case, the calculations that are grown as of late spotlight on increasingly broad cases. For our case it might be face in the inclined position or some other bit of the appearances and furthermore it finds the chance of various countenances. Which implies the turn pivot concerning the current eyewitness from the reference of face in a specific? Or on the other hand regardless of whether there is vertical revolution plane then additionally it can understand the reason. In new kind of calculation it is viewed as that the image or video is a variable which implies that diverse condition in them like tone complexity may change its fluctuation. The measure of light may likewise influence. Likewise the situation of the info may differ the yield. Numerous counts realize the face-recognition task as a two way design separation task as beneath:-

Characterize two constants, one for the eye angle proportion to show Blink. A second consistent for the quantity of back to back frames the eye must be beneath the limit for to set off

\section{\#Alarm \\ EYE AR THRESH $=0.3$ \\ EYE_AR_CONSEC_FRAM \\ $\mathrm{ES}=48$}

1. Initialize the edge counter just as a Boolean used to show if the caution is going off.

COUNTER $=0$

ALARM_ON = False

2. Initialize dib's face locator (HOG-based) and afterward make the facial milestone indicator. 
print ("[INFO] stacking facial

milestone predictor...")

detector= dlib.get_frontal_face_detector ()

indicator $=$ dlib.shape_predictor $(\operatorname{args}[$ "shape

predictor"])

3. grab the records of the facial tourist spots for the left and right eye,respectively

(1Start,lEnd) $=$

face_utils.FACIAL_LANDMARKS_IDX S["left eye"]

$(\mathrm{rStart}, \mathrm{rEnd})=$

face_utils.FACIAL_LANDMARKS_IDX S ["right eye"]

4. start the video stream string

print ("[INFO] beginning video stream thread...")

versus $=$ Video Stream $(\mathrm{src}=\operatorname{args}[$ ["webcam"]).start()

time. Rest (1.0)

start the video stream string The above capacity attracts an obscuration the picture for the relating corner focuses the other boundary are for drawing shading and thickness and sort of lines in the shroud.

\subsection{Recognition of Face Region}

To identify the region of face in the wake of limiting the foundation additional segment from the picture, naming technique is utilized. In the naming strategy, parts are associated in 2-D double picture. This capacity will restore a network of a similar size as parallel picture. It contains marks for the associated objects in parallel picture. it can have an estimation of either 419 where 4 determines 4associated items or 8 where 8 indicates 8 -associated objects. In the event that the contention is overlooked, it defaults to 8. The components of framework are whole number qualities more prominent than or equivalent to 0 . The pixels named 0 are the foundation. The pixels marked 1 make up one item; the pixels named 2 make up a subsequent article, etc.

After that it is required to gauge the properties of picture regions. The region properties work is utilized. This capacity gauges a lot of properties for each named region in the mark network. Positive number components of network relate to various regions. For instance, the arrangement of components of framework equivalent to 1 compares to region 1; the arrangement of components of network equivalent to 2 relates to region 2 , etc. The arrival esteem is a structure exhibit of length lattice. The fields of the structure exhibit signify various estimations for every region, as indicated by properties. Properties can be a comma-isolated rundown of strings, a phone cluster containing strings, the single string 'all', or the string 'essential'. There are set of legitimate property strings. Property strings are case obtuse. This square shape box is utilized to store distinctive worked pictures. What's more, these worked pictures are three sort of. First worked picture which is put away as lattice in square shape box is trimmed cb-cr picture. Second picture is dark picture which is changed over from shading picture. Third picture is histogram evening out picture which is put away as grid in square shape box.

\subsection{Eye Detection}

In our strategy eye is the choice boundary for finding the condition of driver. In spite of the fact that detection of eye might be simpler to find, yet it's actually very confounded. Now it plays out the detection of eye in the necessary specific district with the utilization of detection of a few highlights. For the most part Eigen approach is utilized for this procedure. It is a period taking procedure. At the point when eye detection is done then the outcome is coordinated with the reference or limit an incentive for choosing the condition of the driver. Helpless differentiation of eyes for the most part makes bunches of issues in its detection. After fruitful detection of face eye should be distinguished for additional processing. In our technique eye is the choice boundary for finding the condition of driver. In spite of the fact that detection of eye doesn't look complex yet the real procedure is very chaotic. For this situation it plays out the detection of eye in the predefined district with the utilization of highlight detection. For the most part Eigen approach is utilized for this procedure. It is a period taking procedure. At the point when eye detection is done then the outcome is coordinated with the reference or limit an incentive for choosing the condition of the driver.

\subsection{Digital Image Processing}

Digital image processing is the utilization of PC calculations to perform image processing on digital images. In the field of digital sign processing, there are numerous favorable circumstances of digital image processing with contrast with simple image processing. There is an a lot more extensive scope of calculations to be applied to the info information. It can maintain a strategic distance from issues like the development of commotion and sign distortion during processing. Since images are characterized more than two measurements, even applied on account of more measurements likewise, digital image processing might be displayed as multidimensional frameworks.

Digital image processing licenses the utilization of considerably more troublesome calculations. Digital image processing can offer both increasingly entangled execution at straightforward assignments, and the usage of strategies which would be unimaginable by simple methods. Digital image processing is the main pragmatic innovation for grouping, design acknowledgment, projection, include extraction and multi-scale signal investigation Some strategies which are utilized in digital image processing names as pixlation, head segments examination, direct sifting, anisotropic dispersion, wavelets, neural systems, free segment investigation, shrouded markov model, selfsorting out guides and fractional differential condition. There is important to talk about on Digital Image processing. since all through entire philosophy this method just is utilized. An image might be characterized as a twodimensional capacity, $f(x, y)$, where $x$ and $y$ are spatial directions, and the abundancy of $\mathrm{f}$ at any pair of directions $(\mathrm{x}, \mathrm{y})$ is known as the power or dark degree of the picture by then. When x.y, and the sufficiency estimations of $f$ are generally limited, discrete amounts, the picture is called as an advanced picture. Advanced picture handling alludes to preparing computerized pictures utilizing a computerized 
PC. A propelled picture is made out of a set number of parts, all of which has a particular zone and worth. These parts are implied as picture segments, picture segments, pels, and pixels. Pixel is the term utilized most broadly to signify the components of a computerized picture. Vision is the most developed of among all detects, so clearly pictures take an interest the absolute most imperative job in human recognition. Be that as it may, in contrast to people, who are constrained to the visual band of electromagnetic range, imaging machines spread nearly the whole electromagnetic range. It has run from gamma to radio waves. They can work likewise on pictures created by sources that people don't as a rule relate with pictures. These incorporate electron microscopy, ultrasound, and PC created pictures. Thus, it is effortlessly observed that the computerized picture preparing incorporates a huge, tremendous and wide differed field of uses.

\subsection{Overall Scenario of Implementation}

To defeat the difficult we thought of the arrangement executed as picture preparing. To perform picture handling, OpenCV and dlib open source libraries are utilized. Python is utilized as a language to actualize the thought. A Webcam camera is utilized to ceaselessly follow the facial milestone and development of eyes and lips of the driver. This task for the most part focuses on the tourist spots of lips and eyes of the driver. For discovery of sluggishness, tourist spots of eyes are followed constantly. Pictures are caught utilizing the camera at fix outline pace of 20fps. These pictures are passed to picture handling module which performs face milestone identification to identify interruption and tiredness of driver. In the event that the driver is seen as diverted, at that point a voice (sound) alert and is given and a message is shown on the screen. Following use cases are shrouded in this venture. 1. If eyes of drivers are shut for a limit timeframe then it is viewed as that driver is feeling drowsy and comparing sound caution is utilized to make the driver mindful. 2. If the mouth of driver stays open for the specific timeframe then it is viewed as that driver is yawning and comparing recommendation are given to the driver to conquer sleepiness. 3. If driver don't keep eyes out and about then it is watched utilizing facial milestones, and the relating alert is utilized to make the driver mindful. This usefulness is then actualized with the assistance of python language openCv library, and AI innovation.

\section{$7.1 \quad$ Testing}

To acquire the outcome countless recordings were taken and their precision in deciding eye squints and sluggishness was tried. For this venture, we utilized a webcam associated with the PC. The webcam required white LEDs appended to it for giving better enlightenment. Progressively scenario, infrared LEDs ought to be utilized rather than white LEDs with the goal that the framework is non-meddlesome. Signal is utilized to create ready sound yield to awaken the driver when sluggishness surpasses a specific edge. The framework was tried for various individuals in various encompassing lighting conditions (daytime and evening time). At the point, when the webcam backdrop illumination was turned $\mathrm{ON}$ and the face is kept at an ideal separation, at that point the framework can identify flickers just as languor with over $90 \%$ precision. This is a decent outcome and can be actualized continuously frameworks also. Test yields for different conditions in different pictures are given underneath. Recordings were taken; in which both face and eyes were recognized. In spite of the fact that the two procedures have moderately equivalent exactness. The test was executed on the PC. To confirm the eye divide calculation, the analyzer stood 0.2 to 0.3 away from the Webcam which was situated before the analyzer at an edge with $\pm 15^{\circ}$. The picture of the eye area was separated effectively and was featured by a red square shape. To test the daytime discovery, we controlled the earth light force to be $80 \mathrm{~W}$ and the analyzer remained at a similar identification position as above. At the point, when the analyzer shut the eye for over two seconds, the program demonstrated, "eye shut" at terminal as required. Moreover, when the analyzer squinted the eye, nothing occurred true to form. To test the night identification, the main contrast is that we controlled the encompassing light force to be $20 \mathrm{~W}$. We effectively got a similar discovery result as daytime. The precision for eye flicker was determined by the recipe Precision $=1$ - (complete no. of flickers - no. of flickers recognized)/complete no. of squints. A similar recipe was utilized for figuring precision of sleepiness recognition.

\section{EXPERIMENTAL RESULTS}

To validate our system, we test on driver in the car with real driving condition. We use an camera with buzzer. The results of the eye states are illustrated in image,where the eyes is closed and the system alert the drowsiness and start the alert sound.

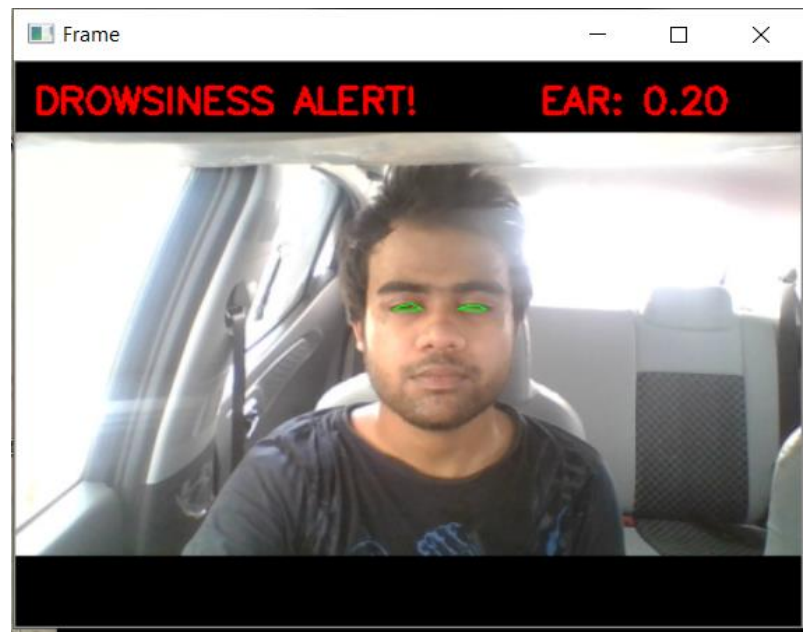

\subsection{CONCLUSION}

In this way we have effectively planned a tiredness recognition framework utilizing Open $\mathrm{Cv}$ Library. With respect to the product part, we satisfied our objective effectively. The recognition calculation could just work successfully and precisely at daytime, yet in addition around evening time. The Eye parcel extraction is smooth and progressively without any postponements on the PC. Also, there is a reward work in the product part - location with 
glasses.Hence, the framework so created was effectively tried.

\subsection{Limitations}

1. Dependence on ambient light:- With helpless lighting conditions despite the fact that face is effectively identified, now and again the framework can't identify the eyes. So it gives an erroneous result which must be dealt with.

Continuously situation infrared backlights ought to be utilized to maintain a strategic distance from helpless lighting conditions.

2. Optimum range requiredWhen the separation among face and webcam isn't at ideal range then certain issues are emerging.

At the point when face is away from the webcam (more than $70 \mathrm{~cm}$ ) at that point the backlight is deficient to light up the face appropriately. So eyes are not identified with high exactness which shows mistake in detection of laziness.

This issue isn't genuinely considered as progressively situation the separation between drivers face and webcam doesn't surpass $50 \mathrm{~cm}$. so the issue never emerges. Considering the above difficulties, the optimum separation go for drowsiness

identification is set to $40-70 \mathrm{~cm}$.

3. Hardware Requirement:- Our framework was run in a PC with a design $2.2 \mathrm{GHz}$ and $2 \mathrm{~GB}$ RAM Pentium double center processor. In spite of the fact that the framework runs fine on higher designs, when a framework has an inferior design, the framework may not be smooth and drowsiness discovery will be moderate. The issue was settled by using committed equipment progressively applications, so there are no issues of casing buffering or more slow identification.

4. Delay in sounding alarm:- When drowsiness level surpasses a certain limit, an alarm is delivered by a framework speaker. It requires a media player to run the sound record. There is a noteworthy deferral between when drowsiness is recognized and when the media player begins and creates the alarm. Be that as it may, progressively, drowsiness is a continuous wonder as opposed to a coincidental event. So

the deferral isn't unreasonably hazardous.

5. Orientation of face:- when the face is inclined to a limited degree it tends to be distinguished, however past this our framework neglects to recognize the face. So when the face isn't recognized, eyes are likewise not detected.This issue is settled by utilizing following capacities which track any development and turn of the items in a picture. A prepared classifier for inclined face and inclined eyes can likewise be utilized to keep away from this sort of issue.

6. Problem with multiple faces If more than one face is identified by the webcam, at that point our framework gives an incorrect outcome. This issue isn't significant as we need to recognize the laziness of a solitary driver.

\subsection{Future Enhancement}

1. Use OpenGL to control the frame rate more accurately

2. To achieve a higher accuracy at night

3. Use bash script to enable our program to auto start after booting

4. Use parallel programming and multi thread to handle image capturing, sending control signal, and running algorithm separately.

5. Use parallel programming such as CUDA to make code faster and more efficient.

\subsection{Future Scope of Application}

This project can be implemented in the form of mobile application to reduce the Cost of hardware.In the real time driver fatigue detection system it is required to slow down a vehicle automatically when fatigue level crosses a certain limit. Instead of threshold drowsiness level it is suggested to design a continuous scale driver fatigue detection system. It monitors the level of drowsiness continuously and when this level exceeds a certain value a signal is generated which controls the hydraulic braking system of the vehicle.

Hardware Components Required:-

Devoted hardware for picture obtaining preparing and show. Interface support with the water driven slowing mechanism which incorporates transfer, clock, stepper engine and a straight actuator.

\section{FUNCTION}

At the point, when laziness level surpasses a specific cutoff then a sign is created which is imparted to the hand-off through the equal port(parallel information move required for quicker results). The hand-off drives the on postpone clock and this clock thus runs the stepper engine for a positive timeframe. The stepper engine is associated with a direct actuator. The straight actuator changes over rotational development of stepper engine to direct movement. This straight movement is utilized to drive a pole which is legitimately associated with the water powered stopping the mechanism of the vehicle. At the point, when the pole moves it applies the brake and the vehicle speed decline Right now there isn't change in zoom or course of the camera during activity. Future work might be to naturally focus on the eyes once they are restricted. This would stay away from the exchange off between having a wide field of view to find the eyes, and a limited view to distinguish weakness. This framework just ganders at the quantity of back to back edges where the eyes are shut. By then it might be past the point where it is possible to give the notice. By examining eye development designs, it is conceivable to discover a strategy to produce the notice sooner. Utilizing 3D pictures is another chance in finding the eyes. The eyes are the most profound piece of a 3D picture, and this perhaps a progressively powerful method of limiting the eyes. 
Versatile linearization is an expansion that can help make the framework progressively powerful. This may likewise dispose of the requirement for the commotion evacuation function, chopping down the calculations expected to discover the eyes. This will likewise permit flexibility to changes in surrounding light. The framework doesn't work for darker looking people. This can be remedied by having a versatile light source. The versatile light source would quantify the measure of light being reflected. On the off chance that little light is being mirrored, the force of the light is expanded. Darker cleaned singular need significantly more light, with the seek that when the twofold picture is built, the face is white, and the foundation is dark.

\section{REFERENCES}

[1] B. Bhowmick and C. Kumar, "Detection and Classification of Eye State in IR Camera for Driver Drowsiness Identification", in Proceeding of the IEEE International Conference on Signal and Image Processing Applications, 2009Bhowmick et Kumar [6] use the Otsu thresholding [7] to extract face region.

[2] OpenCV. Open Source Computer Vision Library Reference Manual, 2001They used the Haar algorithm to detect objects [2] and face classifier implemented by [3] in OpenCV [4] libraries

[3] Adrian Rosebrock, "Object detection with deep learning and OpenCV", pyimagesearch.

[4] Mohana and H. V. R . Aradhya, "Elegant and effici ent algorit hms for real time object detection, counting and classification for video surveillance applications from single fixed camera," 2016 International Conference on Circuits, Controls, Communications and Computing (I4C), Bangalore, 2016, pp. $1-$ 7.

[5] P. Viola, M. Jones, "Robust Real-Time Face Detection", International Journal of Computer Vision 57(2), 137154, 2004

[6] R. Gonzalez and R. Woods, "Digital Image Processing", PearsonEducation, 3rd Edition, 2008.

[7] M. Cerny, M. Dobrovolny, "Eye Tracking System on Embedded Platform", International conference of Applied Electronics 2012

[8] P. Aby, A. Jose, et al, "Implementation and optimization of an Embedded Face Detection system", International Conference on Signal Processing 2011.

[9] G. Bradski and, A. Kaehler, "Learning OpenCV", OReilly Publications, 2008

[10] D. Albanese, G. Merler, S.and Jurman, and R. Visintainer MLPy: high-performance python pack-age for predictive modeling. In NIPS, MLOSS Workshop, 2008.

[11] P.F. Dubois, editor. Python: Batteries Included, volume 9 of Computing in Science \& Engineering.IEEE/AIP, May 2007

[12] S. Van der Walt, S.C Colbert, and G. Varoquaux. The NumPy array: A structure for efficientnumerical computation. Computing in Science and Engineering, 11, 2011

[13] .T. Zito, N. Wilbert, L. Wiskott, and P. Berkes. Modular toolki for data processing (MDP) Pythondata processing framework. Frontiers in Neuroinformatics, 2, 2008.

[14] A. Majumder, L. Behera, and V. K. Subramanian, "Automatic facial expression recognition system using deep network-based data fusion," IEEE Trans. Cybern., vol. 48, pp. 103-114, 2018.

[15] P. F. Felzenszwalb, R. B. Girshick, D. Mcallester, and D. Ramanan, "Object detection with discriminatively trained partbased models," IEEE Trans. Pattern Anal. Mach. Intell., vol. 32, no. 9 , p. $1627,2010$.

[16] K. K. Sung and T. Poggio, "Example-based learning for viewbased human face detection," IEEE Trans. Pattern Anal. Mach. Intell., vol. 20, no. 1, pp. 39-51, 2002

[17] A. Dundar, J. Jin, B. Martini, and E. Culurciello, "Embedded streaming deep neural networks accelerator with applications," IEEE Trans. Neural Netw. \& Learning Syst., vol. 28, no. 7, pp. 1572-1583, 2017

[18] D. Ribeiro, A. Mateus, J. C. Nascimento, and P. Miraldo, “A real-time pedestrian detector using deep learning for humanaware navigation," arXiv:1607.04441, 2016
[19] T.-Y. Lin, P. Dollar, R. B. Girshick, K. He, B. Hariharan, and S J. ' Belongie, "Feature pyramid networks for object detection,' in CVPR, 2017

[20] J. R. Uijlings, K. E. Van De Sande, T. Gevers, and A. W. Smeulders, "Selective search for object recognition," Int. J. of Comput. Vision, vol. 104, no. 2, pp. 154-171, 2013

[21] A. Khan, B. Rinner, and A. Cavallaro, "Cooperative robots to observe moving targets: Review," IEEE Trans. Cybern., vol. 48, pp. 187-198, 2018

[22] V. Marco, What Is Machine Learning? A Definition, 2017.

[23] Technopedia, What Is Computer Vision? - Definition from Techopedia, 2019

[24] H. Kuseto gullari and T. Celik, "Real time vehicle detection and driver warning system using computer vision," 2010.

[25] A. Cheddad, H. Kusetogullari, and H. Grahn, "Object recognition using shape growth pattern," in Proceedings of the 10th International Symposium on Image and Signal Processing and Analysis. IEEE, 2017

[26] N. Sebe, I. Cohen, A. Garg, and T. S. Huang, Machine learning in computer vision. Springer Science \& Business Media, 2005

[27] D. Maturana and S. Scherer, "Voxnet: A 3d convolutional neural network for real-time object recognition," in 2015 IEEE/RSJ International Conference on Intelligent Robots and Systems (IROS). IEEE, 2015

[28] S. Vitabile, A. Paola and F. Sorbello, "Bright Pupil Detection in an Embedded, Real-time Drowsiness Monitoring System", in 24th IEEE International Conference on Advanced Information Networking and Applications, 2010

[29] B. Bhowmick and C. Kumar, "Detection and Classification of Eye State in IR Camera for Driver Drowsiness Identification", in Proceeding of the IEEE International Conference on Signal and Image Processing Applications, 2009

[30] Z. Tian et H. Qin, "Real-time Driver's Eye State Detection", in Proceedings of the IEEE International Conference on Vehicular Electronics and Safety, October 2005

[31] N. Otsu, "A Threshold Selection Method from Gray-Level Histograms", IEEE Transactions on Systems,Man and Cybernatics, pp. 62-66, 1979Bhowmick et Kumar [6] use the Otsu thresholding [7] to extract face region

[32] A. R. Beukman, G. P. Hancke, B. J. Silva, "A multi-sensor system for detection of driver fatigue", Industrial Informatics (INDIN) 2016 IEEE 14th International Conference on, pp. 870 873,2016 